UDC 519.004.942

DOI: $10.20535 /$ SRIT.2308-8893.2020.1.05

\title{
NUMERICAL SIMULATION OF GRAVITATIONAL WAVES FROM A BLACK HOLE, USING CURVATURE TENSORS
}

\author{
Y. MATSUKI, P.I. BIDYUK
}

\begin{abstract}
In this research we formulated the curvature tensors with the system of spherical polar coordinates, which describe the gravitational field and gravitational waves of a black hole; and then we calculated eigenvalues of the curvature tensors to estimate the relative strengths of their components to the stress-energy tensor in Einstein's field equation. For this simulation, we assumed that the time and the distance interact with each other if we travel from Earth to the inside of the black hole, and then the result of the simulation showed that the gravitational waves carry the same components of the gravitational field of the black hole. On the other hand, when we assumed that the time and the distance are independent, which resembles the situation outside of the boundary of the black hole toward Earth, the curvature tensors are different between those of the gravitational field and the gravitational waves. Upon the results of the simulation we conclude that the gravitational waves that come from the inside of the black hole carry the information of the gravitational field inside of the black hole, if we assume that time and space are dependent each other.
\end{abstract}

Keywords: Gravitational field, gravitational waves, curvature tensor, black hole, spherical polar coordinates.

\section{INTRODUCTION (Theory)}

\section{Curvature tensors of gravitational field and gravitational waves of a black hole}

According to Einstein and Dirac [1], the gravitational field of a black hole is described by the curvature tensors:

$$
R_{\mu v}=\Gamma_{\mu \alpha, v}^{\alpha}-\Gamma_{\mu v, \alpha}^{\alpha}-\Gamma_{\mu v}^{\alpha} \Gamma_{\alpha \beta}^{\beta}+\Gamma_{\mu \beta}^{\alpha} \Gamma_{v \alpha}^{\beta},
$$

where $\Gamma_{\mu \nu}^{\alpha}=g^{\alpha \alpha} \Gamma_{\alpha \mu \nu}=\frac{1}{2} g^{\alpha \alpha}\left(g_{\alpha \mu, v}+g_{\alpha v, \mu}-g_{\mu v, \alpha}\right)$, and $g^{\mu \nu}$ are the fundamental tensors that describe the curvature of the 4-dimensional space.

\section{Outside of black hole}

Gravitational field outside of a black hole. According to Dirac [1], the fundamental tensors, $g^{\mu \nu}$, of gravitational field outside of a black hole in spherical polar coordinates are as follows:

$$
g^{\mu \nu}=\left[\begin{array}{cccc}
1-\frac{2 m}{r} & 0 & 0 & 0 \\
0 & -\left(1-\frac{2 m}{r}\right)^{-1} & 0 & 0 \\
0 & 0 & -r^{2} & 0 \\
0 & 0 & 0 & -r^{2} \sin ^{2} \theta
\end{array}\right]=
$$




$$
=\left[\begin{array}{cccc}
e^{2 v} & 0 & 0 & 0 \\
0 & -e^{-2 \lambda} & 0 & 0 \\
0 & 0 & -r^{2} & 0 \\
0 & 0 & 0 & -r^{2} \sin ^{2} \theta
\end{array}\right],
$$

where $2 m$ is a constant, and $m$ is assumed to be the mass of a black hole; and $g^{\mu \nu}$ are functions of a network of curvilinear coordinates, which provide a geodesic, $d s^{2}=g^{00} d t^{2}+g^{11} d r^{2}+g^{22} d \theta^{2}+g^{33} d \varphi^{2}$, outside of the black hole. And then, the curvature tensors $R_{\mu \nu}$ of gravitational field outside of a black hole in spherical polar coordinates are described as follows:

$$
\begin{aligned}
& R_{00}=\left(-v^{\prime \prime}+\lambda^{\prime} v^{\prime}-v^{\prime 2}-\frac{2 \lambda^{\prime}}{r}\right) e^{2 v-2 \lambda}, R_{11}=v^{\prime \prime}-\lambda^{\prime} v^{\prime}+v^{\prime 2}-\frac{2 \lambda^{\prime}}{r}, \\
& R_{22}=\left(1+r v^{\prime}-r \lambda^{\prime}\right) e^{-2 \lambda}-1 ; R_{33}=\left\{\left(1+r v^{\prime}-r \lambda^{\prime}\right) e^{-2 \lambda}-1\right\} \sin ^{2} \theta,
\end{aligned}
$$

while the rest of $R_{\mu v}$ are all zero. Here, $v$ and $\lambda$ are functions of $r$, and $v^{\prime}=\frac{d v}{d r}, v^{\prime \prime}=\frac{d^{2} v}{d r^{2}}$, and $\lambda^{\prime}=\frac{d \lambda}{d r}$

Gravitational waves outside of a black hole. The gravitational waves in curvature tensors are described by the equations:

$$
\begin{gathered}
g^{\mu \nu}{ }_{\rho \sigma, \mu \nu}+g_{, \sigma}^{\mu v}\left(g_{\rho \mu, \nu}-\frac{1}{2} g_{\mu v, \rho}\right)+g_{, \rho}^{\mu \nu}\left(g_{\sigma \mu, v}-\frac{1}{2} g_{\mu v, \sigma}\right)+ \\
+\frac{1}{2} g^{\rho \mu} g_{\beta \beta} g_{\rho \mu, \beta} g_{v \mu, \beta \sigma}+\frac{1}{2} g^{\rho \mu} g_{\beta \beta} g_{\rho \mu, \beta \sigma} g_{v \mu, \beta}+ \\
+\frac{1}{2} g_{, \sigma}^{\rho \mu} g_{\beta \beta} g_{\rho \mu, \beta} g_{v \mu, \beta}+\frac{1}{2} g^{\rho \mu} g_{\beta \beta, \sigma} g_{\rho \mu, \beta} g_{v \mu, \beta}+ \\
+\frac{1}{2} g^{\sigma \mu} g_{\beta \beta} g_{\sigma \mu, \beta} g_{v \mu, \beta \rho}+\frac{1}{2} g^{\sigma \mu} g_{\beta \beta} g_{\sigma \mu, \beta \rho} g_{v \mu, \beta}+ \\
+\frac{1}{2} g_{, \rho}^{\sigma \mu} g_{\beta \beta} g_{\sigma \mu, \beta} g_{v \mu, \beta}+\frac{1}{2} g^{\sigma \mu} g_{\beta \beta, \rho} g_{\sigma \mu, \beta} g_{v \mu, \beta} .
\end{gathered}
$$

Note. In Dirac's original article [1], only the first term , $g_{\rho \sigma, \mu \nu}^{\mu \nu}$, is discussed, but here in our article we also consider those terms from the second to the $12^{\text {th }}$, which were neglected in [1].

If $\beta=\rho, \quad g^{\rho \mu} g_{\beta \beta} g_{\rho \mu, \beta} g_{v \mu, \beta \sigma}=g^{\rho \mu} g_{\rho \rho} g_{\rho \mu, \rho} g_{v \mu, \rho \sigma} ; \quad g^{\rho \mu} g_{\rho \rho}=g_{\rho}^{\mu}=1 \quad$ if $\mu=\rho ; \quad g^{\rho \mu} g_{\rho \rho}=g_{\rho}^{\mu}=0 \quad$ if $\quad \mu \neq \rho ; \quad g_{, \sigma}^{\rho \mu} g_{\beta \beta} \neq 0 \quad$ if $\mu=\rho=\beta ; \quad$ otherwise, $g_{, \sigma}^{\rho \mu} g_{\beta \beta}=0$; and $g^{\rho \mu} g_{\beta \beta, \sigma} \neq 0$ if $\mu=\rho=\beta$; otherwise, $g^{\rho \mu} g_{\beta \beta, \sigma}=0$. Therefore, many terms become zero, and only the following terms remain:

$$
g^{00} g_{00,11}=\left(1-\frac{2 m}{r}\right)\left\{\left(1-\frac{2 m}{r}\right)^{-1}\right\}_{, r r}=\left(1-\frac{2 m}{r}\right)\left\{-2 m \frac{1}{r^{2}}\left(1-\frac{2 m}{r}\right)^{-2}\right\}=
$$




$$
\begin{gathered}
=2\left(1-\frac{2 m}{r}\right)^{-2}\left(\frac{2 m}{r^{2}}\right)+\left(1-\frac{2 m}{r}\right)^{-1} \frac{4 m}{r^{3}} ; \\
g^{11} g_{11,11}=-\left(1-\frac{2 m}{r}\right)^{-1}\left\{-\left(1-\frac{2 m}{r}\right)\right\}_{, r r}=\frac{-4 m}{r^{2}(r-2 m)} ; \\
g^{22} g_{22,11}=\left(-r^{2}\right)\left(-r^{2}\right)_{, r r}=-r^{2}\left(-2 r^{-3}\right)_{, r}=6 r^{2} r^{-4}=\frac{6}{r^{2}} ; \\
g^{33} g_{33,11}=\left(-r^{2} \sin ^{2} \theta\right)\left(-r^{-2} \sin ^{-2} \theta\right)_{, r r}= \\
=-r^{2} \sin ^{2} \theta\left(-2 r^{-3}\right)_{, r} \sin ^{-2} \theta=6 r^{2} r^{-4}=\frac{6}{r^{2}} ; \\
g^{33} g_{33,22}=\left(-r^{2} \sin ^{2} \theta\right)\left(-r^{-2} \sin ^{-2} \theta\right)_{, \theta \theta}=\left(-r^{2} \sin ^{2} \theta\right)\left(2 r^{-2} \sin ^{-3} \theta \cos \theta\right)_{, \theta}= \\
=-2 \sin ^{2} \theta(3 \cos \theta-1) ; \\
g_{, 1}^{11}\left(g_{11,1}-\frac{1}{2} g_{11,1}\right)+g_{, 1}^{11}\left(g_{11,1}-\frac{1}{2} g_{11,1}\right)=\frac{-4 m}{r^{4}-4 r^{3} m+4 r^{2} m^{2}} ; \\
\frac{1}{2} g^{11} g_{11} g_{11,1} g_{11,1}+\frac{1}{2} g^{11} g_{11} g_{11,1} g_{11,1}=\frac{-8 m^{2}}{r^{3}} ; \\
\frac{1}{2} g_{, 1}^{11} g_{11} g_{11,1} g_{11,1}+\frac{1}{2} g_{, 1}^{11} g_{11} g_{11,1} g_{11,1}=\frac{2 m r-4 m^{2}}{r^{3}} ; \\
\frac{1}{2} g^{11} g_{11,1} g_{11,1} g_{11,1}+\frac{1}{2} g^{11} g_{11,1} g_{11,1} g_{11,1}=\frac{8 m^{3}}{r^{5}(r-2 m)},
\end{gathered}
$$

where $(*)_{, r}=\frac{\partial(*)}{\partial r}$ and $(*)_{, r r}=\frac{\partial^{2}(*)}{\partial r^{2}}$; and, $(*)$ is any given function.

\section{Penetrating the boundary of a black hole}

As shown in the equation (1), there is a singularity at $r=2 m$ in one of the curvature tensors, $R_{11}$, which means the presence of the boundary of a black hole, and we cannot see the inside, where $r<2 m$, from the outside, where $r>2 m$. In order to look inside of the black hole, Dirac [1] invented different coordinate system, assuming that the time and space are dependent, by $\tau=t+f(r)$; and, $\rho=t+g(r)$, where $t$ is time, and $r$ is distance. The steps given below show how Dirac [1] described the system of coordinates that extends to the inside of a black hole.

At first, the geodesic in the spherical polar coordinates is defined as follows: $d s^{2}=g^{00} d t^{2}+g^{11} d r^{2}+g^{22} d \theta^{2}+g^{33} d \varphi^{2}$; but, if $t$ and $r$ are dependent on each other, the first two terms, about time and distance, change to the following: 


$$
\begin{aligned}
\left(1-\frac{2 m}{r}\right) d t^{2} & -\left(1-\frac{2 m}{r}\right)^{-1} d r^{2}=d \tau^{2}-\frac{2 m}{r} d \rho^{2}=\left(d t+f^{\prime} d r\right)^{2}-\frac{2 m}{r}\left(d t+g^{\prime} d r\right)^{2}= \\
& =d t^{2}+2 f^{\prime} d t d r+f^{\prime 2} d r^{2}-\frac{2 m}{r}\left(d t^{2}+2 g^{\prime} d t d r+g^{\prime 2} d r^{2}\right)= \\
& =\left(1-\frac{2 m}{r}\right) d t^{2}+2\left(f^{\prime}-\frac{2 m}{r} g^{\prime}\right) d t d r+\left(f^{\prime 2}-\frac{2 m}{r} g^{\prime 2}\right) d r^{2}
\end{aligned}
$$

where $f^{\prime}=\frac{\partial f}{\partial r}$ and $g^{\prime}=\frac{\partial g}{\partial r}$.

The necessary conditions for satisfying $\left(1-\frac{2 m}{r}\right) d t^{2}-\left(1-\frac{2 m}{r}\right)^{-1} d r^{2}=$ $=d \tau^{2}-\frac{2 m}{r} d \rho^{2}$ are:

$$
2\left(f^{\prime}-\frac{2 m}{r} g^{\prime}\right) d t d r=0, f^{\prime}=\frac{2 m}{r} g^{\prime} \text { and } \frac{2 m}{r} g^{\prime 2}-f^{\prime 2}=\left(1-\frac{2 m}{r}\right)^{-1} .
$$

And then, $\frac{2 m}{r} g^{\prime 2}-\left(\frac{2 m}{r} g^{\prime}\right)^{2}=g^{\prime 2} \frac{2 m}{r}\left(1-\frac{2 m}{r}\right)=\left(1-\frac{2 m}{r}\right)^{-1} ; \quad$ then $g^{\prime 2}=\frac{r}{2 m}\left(1-\frac{2 m}{r}\right)^{-2}$, and then $g^{\prime}=\left(\frac{r}{2 m}\right)^{\frac{1}{2}}\left(1-\frac{2 m}{r}\right)^{-1}$.

Now, assume that $r=y^{2}$ and, $2 m=a^{2}$. If $r>2 m, y^{2}>a^{2}$, then $y>a$ :

$$
\begin{gathered}
\frac{d g}{d r}=g^{\prime}=\left(\frac{y^{2}}{a^{2}}\right)^{\frac{1}{2}}\left(1-\frac{a^{2}}{y^{2}}\right)^{-1}=\left(\frac{y^{2}}{a^{2}}\right)^{\frac{1}{2}}\left(\frac{y^{2}-a^{2}}{y^{2}}\right)^{-1}=\frac{y^{2}}{y^{2}-a^{2}} \sqrt{\frac{y^{2}}{a^{2}}}=\frac{y^{2}}{y^{2}-a^{2}} \frac{y}{a} \\
y^{2}=r, \text { so, } \frac{d g}{d y}=\frac{d g}{d r} \frac{d r}{d y}=2 y \frac{d g}{d r}=2 y \frac{y^{3}}{a} \frac{1}{\left(y^{2}-a^{2}\right)}=\frac{2 y^{4}}{a} \frac{1}{y^{2}-a^{2}}
\end{gathered}
$$

Then,

$$
\begin{gathered}
g=\int \frac{2 y^{4}}{a} \cdot \frac{1}{(y+a)(y-a)} d y=\int g^{\prime} \frac{d r}{d y} d y=\int g^{\prime} 2 y d y \\
g=\frac{2}{3 a} y^{3}+2 a y-a^{2} \log \left(\frac{y+a}{y-a}\right) \\
g^{\prime}-f^{\prime}=g^{\prime}-\frac{2 m}{r} g^{\prime}=g^{\prime}\left(1-\frac{2 m}{r}\right)=\left(\frac{r}{2 m}\right)^{\frac{1}{2}}\left(1-\frac{2 m}{r}\right)^{-1}\left(1-\frac{2 m}{r}\right)=\left(\frac{r}{2 m}\right)^{\frac{1}{2}} \\
\frac{2}{3} \frac{1}{\sqrt{2 m}} r^{(3 / 2)}=g-f=\rho-\tau . \\
\text { Thus, } r=\mu(\rho-\tau)^{\frac{2}{3}}, \text { where } \mu=\left(\frac{3}{2} \sqrt{2 m}\right)^{\frac{2}{3}}, \text { and } r=\left(\frac{3}{2} \sqrt{2 m}\right)^{\frac{2}{3}}(\rho-\tau)^{\frac{2}{3}}
\end{gathered}
$$




$$
r^{3 / 2}=(\rho-\tau) \sqrt{2 m} \frac{3}{2}, \text { and } r=\left\{\frac{3}{2} \sqrt{2 m}(\rho-\tau)\right\}^{2 / 3} .
$$

Therefore,

$$
\begin{gathered}
d s^{2}=g^{00} d t^{2}+g^{11} d r^{2}+g^{22} d \theta^{2}+g^{33} d \phi^{2} \\
=\left(1-\frac{2 m}{r}\right) d t^{2}-\left(1-\frac{2 m}{r}\right)^{-1} d r^{2}-r^{2} d \theta^{2}-r^{2} \sin ^{2} \theta d \varphi= \\
=d \tau^{2}-\frac{2 m}{\mu(\rho-\tau)^{2 / 3}} d \rho^{2}-\mu^{2}(\rho-\tau)^{3 / 4} d \theta^{2}-\mu^{2}(\rho-\tau)^{4 / 3} \sin ^{2} \theta d \varphi^{2} .
\end{gathered}
$$

Therefore, the fundamental tensors $g^{\varsigma \eta}$ of gravitational field, which penetrate the boundary of a black hole in spherical polar coordinates, are:

$$
\begin{aligned}
& g^{\varsigma \eta}=\left[\begin{array}{llll}
g^{00} & g^{01} & g^{02} & g^{03} \\
g^{10} & g^{11} & g^{12} & g^{13} \\
g^{20} & g^{21} & g^{22} & g^{23} \\
g^{30} & g^{31} & g^{32} & g^{33}
\end{array}\right]= \\
& =\left[\begin{array}{cccc}
1 & 0 & 0 & 0 \\
0 & -\frac{2 m}{\mu(\rho-\tau)^{2 / 3}} & 0 & 0 \\
0 & 0 & -\mu^{2}(\rho-\tau)^{4 / 3} & 0 \\
0 & 0 & 0 & -\mu^{2}(\rho-\tau)^{4 / 3} \sin ^{2} \theta
\end{array}\right] \text {, }
\end{aligned}
$$

where $g^{\varsigma \eta}$ provide functions of a geodesic, $d s^{2}=g^{00} d t^{2}+g^{11} d r^{2}+g^{22} d \theta^{2}+$ $+g^{33} d \varphi^{2}$, which penetrates the boundary of the black hole.

\section{THE CURVATURE TENSORS FOR NUMERICAL SIMULATION}

Table 1 and Table 2 show the curvature tensors of gravitational field and gravitational waves, which we made for our numerical simulation. These tensors don't have the mathematical singularity; therefore we can simulate the inside of the black hole.

The curvature tensors of gravitational field are:

$$
R_{\varsigma \eta}=\Gamma_{\zeta \alpha, \eta}^{\alpha}-\Gamma_{\varsigma \eta, \alpha}^{\alpha}-\Gamma_{\varsigma \eta}^{\alpha} \Gamma_{\alpha \beta}^{\beta}+\Gamma_{\varsigma \beta}^{\alpha} \Gamma_{\eta \alpha}^{\beta} .
$$

In our simulation, we included the component of, $-\Gamma_{\varsigma \eta}^{\alpha} \Gamma_{\alpha \beta}^{\beta}+\Gamma_{\varsigma \beta}^{\alpha} \Gamma_{\eta \alpha}^{\beta}$, which Dirac [1] neglected.

For example, for $\varsigma=\eta=0, \alpha=2$ and $\beta=1$ we have:

$$
R_{00}=\Gamma_{02,0}^{2}-\Gamma_{00,2}^{2}-\Gamma_{00}^{2} \Gamma_{21}^{1}+\Gamma_{01}^{2} \Gamma_{02}^{1}=
$$




$$
\begin{gathered}
=g^{22} \Gamma_{202,0}-g^{22} \Gamma_{200,2}-g^{22} \Gamma_{200} g^{11} \Gamma_{121}+g^{22} \Gamma_{201} g^{11} \Gamma_{102}= \\
=\frac{1}{2}\left\{-\mu^{2}(\rho-\tau)^{4 / 3}\right\}\left\{-\frac{4}{3}(-1)(\rho-\tau)^{(-7 / 3)}\right\}_{, \tau}=\left\{\frac{-2}{3(\rho-\tau)}\right\}_{, \tau}=\frac{2}{3(\rho-\tau)^{2}},
\end{gathered}
$$

where $\{*\}_{, \tau}=\frac{\partial\{*\}}{\partial \tau}$ and $\{*\}_{, \tau \tau}=\frac{\partial^{2}\{*\}}{\partial \tau^{2}}$, and $\{*\}$ is any given function.

Table 1. Curvature tensors of gravitational field, which extends beyond the

\begin{tabular}{|c|c|c|c|c|c|}
\hline \begin{tabular}{|l|} 
Para- \\
meter
\end{tabular} & $R_{00}$ & $R_{01}=R_{10}$ & $R_{11}$ & $R_{22}$ & $R_{33}$ \\
\hline \multirow{2}{*}{$\begin{array}{l}\alpha=0 \\
\beta=0\end{array}$} & & \multirow[b]{2}{*}{0} & $-\mu$ & 14 & -14 \\
\hline & & & $\overline{6 m(\rho-\tau)^{4 / 3}}$ & $\overline{9 \mu^{2}(\rho-\tau)^{10 / 3}}$ & $\overline{9 \mu^{2}(\rho-\tau)^{10 / 3} \sin ^{2} \theta}$ \\
\hline \multirow{2}{*}{$\beta=1$} & \multirow{2}{*}{0} & \multirow[b]{2}{*}{ U } & $\mu$ & 4 & -4 \\
\hline & & & $\overline{6 m(\rho-\tau)^{4 / 3}}$ & $\overline{3 \mu^{2}(\rho-\tau)^{10 / 3}}$ & $3 \mu^{2}(\rho-\tau)^{10 / 3} \sin ^{2} \theta$ \\
\hline \multirow{2}{*}{$\beta=2$} & \multirow{2}{*}{0} & \multirow[b]{2}{*}{0} & $5 \mu$ & 22 & -2 \\
\hline & & & $\overline{18 m(\rho-\tau)^{4 / 3}}$ & $\overline{9 \mu^{2}(\rho-\tau)^{10 / 3}}$ & $\overline{\mu^{2}(\rho-\tau)^{10 / 3} \sin ^{2} \theta}$ \\
\hline \multirow{2}{*}{$\beta=3$} & \multirow[b]{2}{*}{0} & \multirow[t]{2}{*}{0} & $5 \mu$ & 2 & -14 \\
\hline & & & $\overline{18 m(\rho-\tau)^{4 / 3}}$ & $\overline{\mu^{2}(\rho-\tau)^{10 / 3}}$ & $\overline{9 \mu^{2}(\rho-\tau)^{10 / 3} \sin ^{2} \theta}$ \\
\hline \multirow{2}{*}{$\begin{array}{l}\alpha=1, \\
\beta=0\end{array}$} & -1 & \multirow[b]{2}{*}{0} & $\mu$ & $4 m$ & $4 m$ \\
\hline & $\overline{3(\rho-\tau)^{2}}$ & & $\overline{18 m(\rho-\tau)^{4 / 3}}$ & $\overline{\mu^{2}(\rho-\tau)^{4}}$ & $\overline{\mu^{3}(\rho-\tau)^{4} \sin ^{2} \theta}$ \\
\hline \multirow{2}{*}{$\beta=1$} & -2 & \multirow[b]{2}{*}{0} & \multirow{2}{*}{0} & $32 m$ & $32 m$ \\
\hline & $\overline{9(\rho-\tau)^{2}}$ & & & $\overline{9 \mu^{2}(\rho-\tau)^{4}}$ & $\overline{9 \mu^{3}(\rho-\tau)^{4} \sin ^{2} \theta}$ \\
\hline \multirow{2}{*}{$\beta=2$} & -1 & -2 & 2 & $4 m$ & $44 m$ \\
\hline & $\overline{3(\rho-\tau)^{2}}$ & $\overline{9(\rho-\tau)^{2}}$ & $\overline{9(\rho-\tau)^{2}}$ & $\overline{\mu^{2}(\rho-\tau)^{4}}$ & $\overline{9 \mu^{3}(\rho-\tau)^{4} \sin ^{2} \theta}$ \\
\hline \multirow{2}{*}{$\beta=3$} & -1 & -2 & 2 & $44 m$ & $4 m$ \\
\hline & $\overline{3(\rho-\tau)^{2}}$ & $\overline{9(\rho-\tau)^{2}}$ & $\overline{9(\rho-\tau)^{2}}$ & $\overline{9 \mu^{2}(\rho-\tau)^{4}}$ & $\overline{\mu^{3}(\rho-\tau)^{4} \sin ^{2} \theta}$ \\
\hline \multirow{2}{*}{$\begin{aligned} \alpha & =2, \\
\beta & =0\end{aligned}$} & 2 & -2 & 2 & 4 & $1+3 \cot ^{2} \theta$ \\
\hline & $3(\rho-\tau)^{2}$ & $3(\rho-\tau)^{2}$ & $3(\rho-\tau)^{2}$ & $9 \mu^{2}(\rho-\tau)^{10 / 3}$ & $\sin ^{2} \theta$ \\
\hline \multirow{2}{*}{$\beta=1$} & 2 & -2 & 2 & $-8 m$ & $1+3 \cot ^{2} \theta$ \\
\hline & $\overline{3(\rho-\tau)^{2}}$ & $\overline{3(\rho-\tau)^{2}}$ & $\overline{3(\rho-\tau)^{2}}$ & $\overline{9 \mu^{2}(\rho-\tau)^{4}}$ & $\sin ^{2} \theta$ \\
\hline \multirow{2}{*}{$\beta=2$} & 10 & -10 & 10 & & $1+3 \cot ^{2} \theta$ \\
\hline & $\overline{9(\rho-\tau)^{2}}$ & $\overline{9(\rho-\tau)^{2}}$ & $\overline{9(\rho-\tau)^{2}}$ & 0 & $\sin ^{2} \theta$ \\
\hline \multirow{2}{*}{$\beta=3$} & 2 & -2 & 2 & & $1+3 \cot ^{2} \theta$ \\
\hline & $\overline{3(\rho-\tau)^{2}}$ & $\overline{3(\rho-\tau)^{2}}$ & $\overline{3(\rho-\tau)^{2}}$ & 0 & $\sin ^{2} \theta$ \\
\hline \multirow{2}{*}{$\begin{array}{l}\alpha=3 \\
\beta=0\end{array}$} & 2 & -2 & 2 & 1 & 4 \\
\hline & $\overline{3(\rho-\tau)^{2}}$ & $\overline{3(\rho-\tau)^{2}}$ & $\overline{3(\rho-\tau)^{2}}$ & $\overline{\sin ^{2} \theta}$ & $\overline{9 \mu^{2}(\rho-\tau)^{10 / 3} \sin ^{2} \theta}$ \\
\hline
\end{tabular}
boundary of a black hole 
Continued Tabl. 1

\begin{tabular}{|c|c|c|c|c|c|}
\hline $\begin{array}{c}\text { Para- } \\
\text { meter }\end{array}$ & $R_{00}$ & $R_{01}=R_{10}$ & $R_{11}$ & $R_{22}$ & $R_{33}$ \\
\hline$\beta=1$ & $\frac{2}{3(\rho-\tau)^{2}}$ & $\frac{-2}{3(\rho-\tau)^{2}}$ & $\frac{2}{3(\rho-\tau)^{2}}$ & $\frac{1}{\sin ^{2} \theta}$ & $\frac{-8 m}{9 \mu^{3}(\rho-\tau)^{4} \sin ^{2} \theta}$ \\
\hline$\beta=2$ & $\frac{2}{3(\rho-\tau)^{2}}$ & $\frac{-2}{3(\rho-\tau)^{2}}$ & $\frac{2}{3(\rho-\tau)^{2}}$ & $\frac{1}{\sin ^{2} \theta}$ & $-\frac{\cot ^{2} \theta}{\sin ^{2} \theta}$ \\
\hline$\beta=3$ & $\frac{10}{9(\rho-\tau)^{2}}$ & $\frac{-10}{9(\rho-\tau)^{2}}$ & $\frac{10}{9(\rho-\tau)^{2}}$ & $\frac{1}{\sin ^{2} \theta}+\cot ^{2} \theta$ & 0 \\
\hline
\end{tabular}

For $R_{02}=R_{20}$, only $-\Gamma_{02}^{2} \Gamma_{23}^{3}=\frac{2 \cot \theta}{3(\rho-\tau)} \quad(\alpha=2, \beta=3)$ and $\Gamma_{03}^{3} \Gamma_{23}^{3}=$ $=\frac{-2 \cot \theta}{3(\rho-\tau)}(\alpha=3, \beta=3)$ remain: therefore $R_{02}=R_{20}=-\Gamma_{02}^{2} \Gamma_{23}^{3}+\Gamma_{03}^{3} \Gamma_{23}^{3}=0$; for $R_{12}=R_{21}$, only $-\Gamma_{12}^{2} \Gamma_{23}^{3}=\frac{-2 \cot \theta}{3(\rho-\tau)}(\alpha=2, \beta=3)$, and $\Gamma_{13}^{3} \Gamma_{23}^{3}=\frac{2 \cot \theta}{3(\rho-\tau)}$ $(\alpha=3, \beta=3)$ remain. Therefore $R_{12}=R_{21}=-\Gamma_{12}^{2} \Gamma_{23}^{3}+\Gamma_{13}^{3} \Gamma_{23}^{3}=0$; and all other components such as $R_{13}, R_{31}, R_{23}$ and $R_{32}$ are 0 . Then, according to Einstein's rule $\left(R_{\mu \nu}=\sum_{\mu \nu} R_{\mu \nu}\right)$ we summate all the components:

$$
\begin{aligned}
R_{\mu \nu}= & \frac{56}{9(\rho-\tau)^{2}}+\frac{11 \mu}{18 m(\rho-\tau)^{\frac{4}{3}}}+\frac{140 m}{9 \mu^{2}(\rho-\tau)^{4}}+\frac{8}{\sin ^{2} \theta}+\cot ^{2} \theta+ \\
& +\frac{140 m}{9 \mu^{3}(\rho-\tau)^{4} \sin ^{2} \theta}+\frac{35 \cot ^{2} \theta}{\sin ^{2} \theta}-\frac{28}{9 \mu^{2}(\rho-\tau)^{\frac{10}{3}} \sin ^{2} \theta} .
\end{aligned}
$$

The curvature tensors of gravitational waves, which penetrate the boundary of a black hole, are:

$$
\begin{aligned}
g^{\varsigma \eta}{ }_{\rho \sigma, \varsigma \eta} & +g_{, \sigma}^{\varsigma \eta}\left(g_{\rho \varsigma, \eta}-\frac{1}{2} g_{\varsigma \eta, \rho}\right)+g_{, \rho}^{\varsigma \eta}\left(g_{\sigma \varsigma, \eta}-\frac{1}{2} g_{\varsigma \eta, \sigma}\right)+ \\
+ & \frac{1}{2} g^{\rho \varsigma} g_{\beta \beta} g_{\rho \varsigma, \beta} g_{\eta \varsigma, \beta \sigma}+\frac{1}{2} g^{\rho \varsigma} g_{\beta \beta} g_{\rho \varsigma, \beta \sigma} g_{\eta \varsigma, \beta}+ \\
& +\frac{1}{2} g_{, \sigma}^{\rho \varsigma} g_{\beta \beta} g_{\rho \varsigma, \beta} g_{\eta \varsigma, \beta}+\frac{1}{2} g^{\rho \varsigma} g_{\beta \beta, \sigma} g_{\rho \varsigma, \beta} g_{\eta \varsigma, \beta}+ \\
+ & \frac{1}{2} g^{\sigma \varsigma} g_{\beta \beta} g_{\sigma \varsigma, \beta} g_{\varsigma \eta, \beta \rho}+\frac{1}{2} g^{\sigma \varsigma} g_{\beta \beta} g_{\sigma \varsigma, \beta \rho} g_{\varsigma \eta, \beta}+ \\
& +\frac{1}{2} g_{, \rho}^{\sigma \varsigma} g_{\beta \beta} g_{\sigma \varsigma, \beta} g_{\eta \zeta, \beta}+\frac{1}{2} g^{\sigma \varsigma} g_{\beta \beta, \rho} g_{\sigma \varsigma, \beta} g_{\eta \varsigma, \beta} .
\end{aligned}
$$


If $\beta=\rho, g^{\rho \varsigma} g_{\beta \beta} g_{\rho \varsigma, \beta \sigma} g_{\eta \varsigma, \beta}=g^{\rho \varsigma} g_{\rho \rho} g_{\rho \varsigma, \rho} g_{\eta \varsigma, \rho \sigma} ; g^{\rho \varsigma} g_{\rho \rho}=g_{\rho}^{\varsigma}=1$ if $\varsigma=\rho$; $g^{\rho \varsigma} g_{\rho \rho}=g_{\rho}^{\varsigma}=0$ if $\varsigma \neq \rho ; g_{, \sigma}^{\rho \varsigma} g_{\beta \beta} \neq 0$ if $\varsigma=\rho=\beta$, otherwise $g_{, \sigma}^{\rho \mu} g_{\beta \beta}=0$; and, $g^{\rho \varsigma} g_{\beta \beta, \sigma} \neq 0$ if $\varsigma=\rho=\beta$, otherwise $g^{\rho \varsigma} g_{\beta \beta, \sigma}=0$.

Table 2. Curvature tensors of gravitational waves, which penetrates the boundary of a black hole

\begin{tabular}{|c|c|}
\hline $\begin{array}{c}\varsigma=\eta=0 \\
\rho=\sigma= \\
=1,2,3 \\
\beta=0,1,2,3\end{array}$ & $\begin{array}{c}g^{00} g_{11,00}=\left\{-\frac{\mu}{2 m}(\rho-\tau)^{2 / 3}\right\}_{, \tau \tau}=\left\{-\frac{\mu}{2 m}(-1) \frac{2}{3}(\rho-\tau)^{-(-1 / 3)}\right\}_{, \tau}= \\
=\frac{\mu}{9 m(\rho-\tau)^{4 / 3}}, g^{00} g_{22,00}=-\frac{28}{9 \mu^{2}(\rho-\tau)^{10 / 3}}, \\
g^{00} g_{33,00}=-\frac{28}{9 \mu^{2}(\rho-\tau)^{10 / 3} \sin ^{2} \theta}\end{array}$ \\
\hline $\begin{array}{c}\varsigma=\eta=1, \\
\rho=\sigma=0, \\
\beta=0,1,2,3\end{array}$ & $-g_{, 0}^{11} g_{11,0}=\frac{4}{9(\rho-\tau)^{2}}$ \\
\hline $\begin{array}{c}\varsigma=\eta=1, \\
\rho=\sigma=1, \\
\beta=0\end{array}$ & $\begin{array}{c}g^{11} g_{11,11}+g_{, 1}^{11}\left(g_{11,1}-\frac{1}{2} g_{11,1}\right)+g_{, 1}^{11}\left(g_{11,1}-\frac{1}{2} g_{11,1}\right)=-\frac{2}{9(\rho-\tau)^{2}}-\frac{4}{9(\rho-\tau)^{2}}, \\
\frac{1}{2} g^{11} g_{00} g_{11,0} g_{11,01}=\frac{-2 \mu}{81 m(\rho-\tau)^{10 / 3}}, \frac{1}{2} g_{, 1}^{11} g_{00} g_{11,0} g_{11,0}=\frac{-\mu}{27 m(\rho-\tau)^{11 / 3}}, \\
\frac{1}{2} g^{11} g_{00,1} g_{11,0} g_{11,0}=\frac{\mu}{27 m(\rho-\tau)^{7 / 3}}, \frac{1}{2} g^{11} g_{00,1} g_{11,0} g_{11,0}=0, \\
\frac{1}{2} g^{11} g_{00} g_{11,0} g_{11,01}=\frac{-\mu}{27 m(\rho-\tau)^{7 / 3}}, \frac{1}{2} g^{11} g_{00} g_{11,01} g_{11,0}=\frac{4 \mu}{108 m(\rho-\tau)^{7 / 3}}, \\
\frac{1}{2} g_{, 1}^{11} g_{00} g_{11,0} g_{11,0}=\frac{2 \mu}{27 m(\rho-\tau)^{7 / 3}}, \frac{1}{2} g^{11} g_{00,1} g_{11,0} g_{11,0}=\frac{-\mu}{9 m(\rho-\tau)^{4 / 3}}\end{array}$ \\
\hline$\beta=1$ & $\begin{array}{l}g^{11} g_{11,11}+g_{, 1}^{11}\left(g_{11,1}-\frac{1}{2} g_{11,1}\right)+g_{, 1}^{11}\left(g_{11,1}-\frac{1}{2} g_{11,1}\right)=-\frac{2}{9(\rho-\tau)^{2}}-\frac{4}{9(\rho-\tau)^{2}}, \\
\frac{1}{2} g^{11} g_{11} g_{11,1} g_{11,11}=\frac{-\mu^{2}}{54 m^{2}(\rho-\tau)^{5 / 3}}, \frac{1}{2} g^{11} g_{11} g_{11,11} g_{11,1}=\frac{-\mu^{2}}{54 m^{2}(\rho-\tau)^{5 / 3}}, \\
\frac{1}{2} g_{, 1}^{11} g_{11} g_{11,1} g_{11,1}=\frac{-\mu^{2}}{27 m^{2}(\rho-\tau)^{5 / 3}}, \frac{1}{2} g^{11} g_{11,1} g_{11,11} g_{11,1}=\frac{\mu^{2}}{27 m^{2}(\rho-\tau)^{5 / 3}}, \\
\frac{1}{2} g^{11} g_{11} g_{11,1} g_{11,11}=\frac{-\mu^{2}}{54 m^{2}(\rho-\tau)^{5 / 3}}, \frac{1}{2} g^{11} g_{11} g_{11,11} g_{11,1}=\frac{-\mu^{2}}{54 m^{2}(\rho-\tau)^{5 / 3}}, \\
\frac{1}{2} g_{, 1}^{11} g_{11} g_{11,1} g_{11,1}=\frac{\mu^{2}}{27 m^{2}(\rho-\tau)^{5 / 3}}, \frac{1}{2} g^{11} g_{11,1} g_{11,11} g_{11,1}=\frac{\mu^{2}}{27 m^{2}(\rho-\tau)^{5 / 3}}\end{array}$ \\
\hline$\beta=2$ & $\begin{array}{c}g^{11} g_{11,11}+g_{, 1}^{11}\left(g_{11,1}-\frac{1}{2} g_{11,1}\right)+g_{, 1}^{11}\left(g_{11,1}-\frac{1}{2} g_{11,1}\right)= \\
=-\frac{2}{9(\rho-\tau)^{2}}-\frac{4}{9(\rho-\tau)^{2}}\end{array}$ \\
\hline
\end{tabular}


Continued Tabl. 2

\begin{tabular}{|c|c|}
\hline$\beta=3$ & $\begin{array}{c}g^{11} g_{11,11}+g_{, 1}^{11}\left(g_{11,1}-\frac{1}{2} g_{11,1}\right)+g_{, 1}^{11}\left(g_{11,1}-\frac{1}{2} g_{11,1}\right)= \\
=-\frac{2}{9(\rho-\tau)^{2}}-\frac{4}{9(\rho-\tau)^{2}}\end{array}$ \\
\hline $\begin{array}{c}\varsigma=\eta=1 \\
\rho=\sigma=2,3 \\
\beta=0,1,2,3\end{array}$ & $g^{11} g_{22,11}=\frac{56 m}{9 \mu^{3}(\rho-\tau)^{4}}, g^{11} g_{33,11}=\frac{56 m}{9 \mu^{3}(\rho-\tau)^{4} \sin ^{2} \theta}$ \\
\hline $\begin{array}{l}\varsigma=\eta=2, \\
\rho=\sigma=0,1,3 \\
\beta=0,1,2,3\end{array}$ & $\begin{array}{c}-g_{, 1}^{22} g_{22,0}=-\frac{16}{9(\rho-\tau)^{2}},-g_{, 1}^{22} g_{22,1}=\frac{16}{9(\rho-\tau)^{2}} \\
g^{22} g_{33,22}=\frac{6 \cos \theta}{\sin ^{4} \theta}+\frac{2}{\sin ^{2} \theta}\end{array}$ \\
\hline $\begin{array}{l}\varsigma=\eta=3 \\
\rho=\sigma=0,1,2 \\
\beta=0,1,2,3\end{array}$ & $\begin{array}{c}-g_{, 0}^{33} g_{33,0}=-4 \frac{\cos ^{2} \theta}{\sin ^{2} \theta}=-4 \cot ^{2} \theta,-g_{, 1}^{33} g_{33,1}=\frac{16}{9(\rho-\tau)^{2}} \\
-g_{, 2}^{33} g_{33,2}=-4 \cot ^{2} \theta\end{array}$ \\
\hline
\end{tabular}

Note. All other components are zero.

Now, we summate all the components:

$$
\begin{gathered}
\frac{4 \mu}{9 m(\rho-\tau)^{4 / 3}}-\frac{112}{9 \mu^{2}(\rho-\tau)^{10 / 3}}-\frac{112}{9 \mu^{2}(\rho-\tau)^{10 / 3} \sin ^{2} \theta}+\frac{-16}{9(\rho-\tau)^{2}}-\frac{2}{3(\rho-\tau)^{2}}- \\
-\frac{2 \mu}{81 m(\rho-\tau)^{10 / 3}}+\frac{\mu}{9 m(\rho-\tau)^{7 / 3}}-\frac{\mu}{9 m(\rho-\tau)^{4 / 3}}-\frac{2}{(\rho-\tau)^{2}}+\frac{224 m}{9 \mu^{3}(\rho-\tau)^{4}}+ \\
+\frac{224 m}{9 \mu^{3}(\rho-\tau)^{4} \sin ^{2} \theta}+\frac{24 \cos \theta}{\sin ^{4} \theta}+\frac{8}{\sin ^{2} \theta}+\frac{64}{9(\rho-\tau)^{2}}-32 \cot ^{2} \theta
\end{gathered}
$$

\section{NUMERICAL SIMULATION}

\section{Algorithm}

Einstein's field equation [1] that rules the motion of particles in the gravitational field is: $\left(R^{\mu v}-\frac{1}{2} g^{\mu v} R\right)_{, v}=0$, where $R$ is a scalar tensor, $R=R_{\mu}^{\mu}=g^{\mu v} R_{\mu v}$, which $\quad$ are $\quad R=R_{0}^{0}=g^{00} R_{00}, \quad R=R_{1}^{1}=g^{11} R_{11}, \quad R=R_{2}^{2}=g^{22} R_{22}, \quad R=R_{3}^{3}=$ $=g^{33} R_{33}$. Then, for example, $R_{00}-\frac{1}{2} g_{00} g^{00} R_{00}=R_{00}-\frac{1}{2} R_{00}=\frac{1}{2} R_{00}$.

Now, we use the relation: $R_{\mu \nu}-\frac{1}{2} g_{\mu \nu} R=k T$, where $T$ is the stress-energy tensor and $k$ is a constant [2]. Then, we set the following algorithm to simulate the relative intensities of the components of curvature tensors.

For example, when $\mu=v=0, R_{00}-\frac{1}{2} g_{00} g^{00} R_{00}=R_{00}-\frac{1}{2} R_{00}=\frac{1}{2} R_{00}$. In our simulation, we omit the factor of, $1 / 2$, because we only use the selected vec- 
tors of the coordinates, which are taken from the obtained curvature tensors in Table 1 for gravitational field and in Table 2 for gravitational waves.

Then, from Table 1 , for example when $\alpha=1$ and $\beta=0, R_{00}=\frac{-1}{3(\rho-\tau)^{2}}$ and when $\alpha=3$ and $\beta=0, R_{22}=\frac{1}{\sin ^{2} \theta}$. Then, for example, if we only summate these two components of $R_{00}$ (when $\alpha=1$ and $\beta=0$ ) and $R_{22}$ (when $\alpha=3$ and $\beta=0$ ), and then, our algorithm is shown as follows:

$$
\begin{gathered}
H=k T-\left(R_{00}+R_{22}\right)=k T-\left\{\frac{-1}{3(\rho-\tau)^{2}}+\frac{1}{\sin ^{2} \theta}\right\}=k T-\left\{a X_{1}+b X_{2}\right\}, \\
H^{2}=\left\{k T-\left(R_{00}+R_{22}\right)\right\}^{2}=\left[k T-\left\{a X_{1}+b X_{2}\right\}\right]^{2},
\end{gathered}
$$

where $a$ and $b$ are constants.

Then, we assume: $\frac{\partial E\left(H^{2}\right)}{\partial a}=E\left(\frac{\partial H^{2}}{\partial a}\right)=2 E\left(H \frac{\partial H}{\partial a}\right)=-2 E\left(X_{1} H\right)=0$, and $\frac{\partial E\left(H^{2}\right)}{\partial b}=E\left(\frac{\partial H^{2}}{\partial b}\right)=2 E\left(H \frac{\partial H}{\partial b}\right)=-2 E\left(X_{2} H\right)=0, \quad$ where $\quad E\left(X_{1} H\right) \quad$ and $E\left(X_{2} H\right)$ are operators to calculate averages of $X_{1} H$ and, $X_{2} H$.

$E\left(X_{1} H\right)=0$ and $E\left(X_{2} H\right)=0$ are equivalent to solving the problem of $C\left(X_{1}, H\right)=0$ and $C\left(X_{2}, H\right)=0$, where $C\left(X_{1}, H\right)$ is the covariance of $X_{1}$ and $H$; and, $C\left(X_{2}, H\right)$ is the covariance of $X_{2}$ and, $H$.

Now, $c=\left[\begin{array}{l}a \\ b\end{array}\right]$, where $a$ and $b$ are the eigenvalues of $R_{00}+R_{22}$, and $X=\left[\begin{array}{ll}X_{1} & X_{2}\end{array}\right] ;$ then $H=k T-\left(R_{00}+R_{22}\right)=k T-X c$, and $X^{\prime} H=0$, then $X^{\prime}(k T-X c)=0$, where $X^{\prime}$ is transpose matrix of $X$. Then, $X^{\prime} X c=X^{\prime} k T$, $c=\left(X^{\prime} X\right)^{-1} X^{\prime} k T=Q^{-1} X^{\prime} k T$, and $\Sigma=V(c)=\sigma^{2} Q^{-1}=\sigma^{2}\left(X^{\prime} X\right)^{-1}$, where $V(c)=\sigma^{2}$ is the variance of $c$, and $\sigma^{2}=e^{\prime} e /(n-l)$, where $e=M \cdot k T$, $M=I-X\left(X^{\prime} X\right)^{-1} X^{\prime}, n$ is a number of rows of each column of $X$ (in this simulation $n=23), l$ is a number of columns of $X, I$ is a $23 \times 23$ unit matrix, $\left(X^{\prime} X\right)^{-1}$ is the inverse matrix of $X^{\prime} X$, and $e^{\prime}$ is the transpose vector of $e$.

By calculating $c$ and, $V(c)$, we can estimate the strength of each component of $\left(R_{00}+R_{22}\right)$ to the stress-energy tensor, in the system of spherical polar coordinates.

We can also expand the size of matrix not only, $l=2$, but also to $l>2$ such as $c^{*}=\left[c_{1}, c_{2}, \ldots, c_{k}\right]$, and $X=\left[X_{1} X_{2} \ldots X_{k}\right]$, where $c^{*}$ is a transposed vector of $c$, so that we were able to calculate not only $\left(R_{00}+R_{22}\right)$, but more general $R_{\mu \nu}$ in our numerical simulation.

\section{Input data}

At first, our time $t$ on Earth is set as shown in Fig. 1 and Fig. 2, with which its slope to the distance $r$ from the center of a black hole toward outside is a con- 
stant, which is consistent with Einstein's theory that the speed of light is a constant. Then, $\tau$ is a relative time in the coordinate system, which expands and shrinks depending on the distance $r$, where $\tau=t+f(r)$; and, $\rho$ is the relative distance, which expands and shrinks depending on the time $t$, where $\rho=t+g(r)$, and $f(r), g(t)$ are conjugate functions of $t$ and $r$. (For the simulation, we assumed case-1: $f(r)=\log r \quad$ and $g(t)=e^{-r} ; \quad$ and, case-2: $f(r)=r \quad$ and $g(t)=(1 / 4))$. According to Dirac [1], the collapse of a star into a black hole would take an infinite time at our clocks on Earth, but it takes only a finite time relatively to the collapsing matter on the star itself. From this Dirac's statement, we assumed that $\tau$ is larger when the relative distance, $\rho$, from the center of the black hole is smaller. Also, in this simulation we set the stress-energy tensor $k T$ to be 1 ; because, the purpose of this simulation is to measure the order of magnitude of the relative strength of each component of $R_{\mu v}$ to the stress-energy tensor.
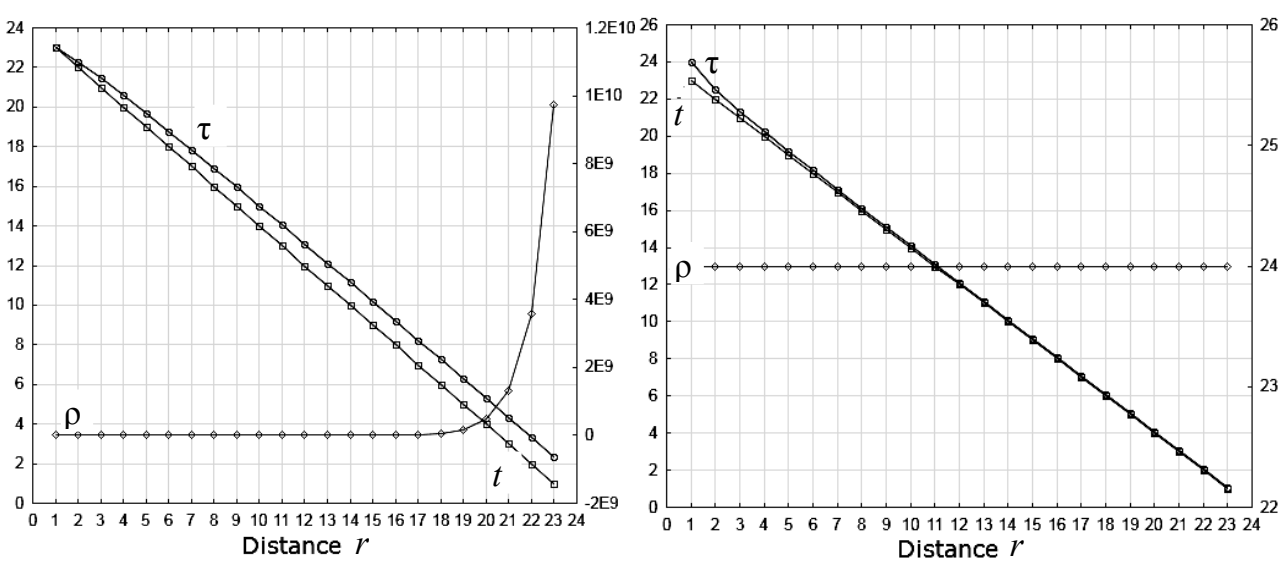

Fig. 1. Input data for simulation Case 1: Fig. 2. Input data for simulation Case 2: $f(r)=\log r$ and $g(t)=e^{-r}$ $f(r)=r$ and $g(t)=1 / 4$

For the simulation of the gravitational waves we assumed the angles, $\theta$, as shown in Fig. 3, as if it becomes smaller in far distance from a black hole; on the
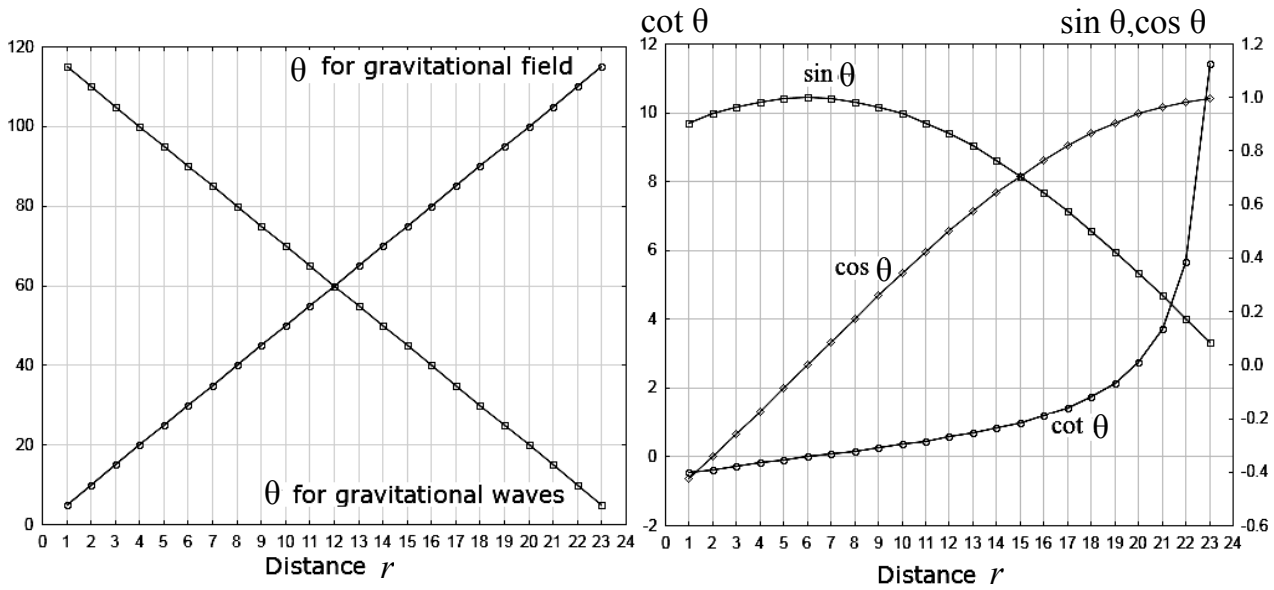

Fig. 3. Angles $\theta$ for simulating gravitational Fig. 4. $\sin \theta, \cos \theta$ and $\cot \theta$ for simulation field and gravitational waves of gravitational waves 
other hand, for the simulation of the spatial expansion of the gravitational field of a black hole, we assumed as if $\theta$ becomes larger in far distance. With this assumption, the gravitational waves of sign cosign and cotangent curves behave like as shown in Fig. 4.

\section{Results}

Table 3 shows the calculated eigenvalues $c$ of $R_{\mu \nu}$ and $\sqrt{V(c)}$ for the gravitational field, and Fig. 5, 6 and Fig. 7 show selected functions that have the negative coefficients, which mean the gravity, and of which each element of $\sqrt{V(c)}$ is smaller than each element of $c$. Table 3 also shows the calculated eigenvalues of the gravitational waves, and Fig. 8 shows the function of $\frac{1}{\sin ^{2} \theta}$ that have the positive coefficients, and of which $\sqrt{V(c)}$ is smaller than its value of $c$. We selected only, $\frac{1}{\sin ^{2} \theta}$, because it has the positive coefficient, assuming that the gravitational waves should give positive impact to the stress-energy tensor, $k T$.

Tab le 3. Results of the simulation of gravitational field and gravitational waves

\begin{tabular}{|c|c|c|c|c|}
\hline \multirow[t]{2}{*}{ Components } & \multicolumn{2}{|c|}{$\begin{array}{c}c \text { and } \sqrt{V(c)} \\
\text { of Gravitational field }\end{array}$} & \multicolumn{2}{|c|}{$\begin{array}{c}c \text { and } \sqrt{V(c)} \\
\text { of Gravitational waves }\end{array}$} \\
\hline & Case-1 & Case-2 & Case-1 & Case-2 \\
\hline$\frac{1}{(\rho-\tau)^{2}}$ & $\begin{array}{c}-1,850 \cdot 10^{-2} \\
(6,298)\end{array}$ & $\begin{array}{l}4,406 \cdot 10^{-4} \\
(0,0002776)\end{array}$ & $\begin{array}{c}5,950 \cdot 10^{-8} \\
\left(-1,059 \times 10^{16}\right)\end{array}$ & $\begin{array}{l}-3,190 \cdot 10^{-3} \\
(0,7698)\end{array}$ \\
\hline$\frac{1}{(\rho-\tau)^{4 / 3}}$ & $\begin{array}{c}-5,750 \cdot 10^{-2} \\
\left(2,060 \cdot 10^{-17}\right)\end{array}$ & $\begin{array}{c}5,118 \cdot 10^{-8} \\
\left(3,211 \cdot 10^{-8}\right)\end{array}$ & $\begin{array}{l}-2,088 \cdot 10^{-12} \\
(0,03356)\end{array}$ & $\begin{array}{l}-5,308 \cdot 10^{-8} \\
\left(1,511 \cdot 10^{-5}\right)\end{array}$ \\
\hline$\frac{1}{(\rho-\tau)^{4}}$ & $\begin{array}{c}-24,58 \\
(6546,0)\end{array}$ & $\begin{array}{c}-6,932 \cdot 10^{-3} \\
(0,002527)\end{array}$ & $\begin{array}{l}6,591 \cdot 10^{-12} \\
\left(2,672 \cdot 10^{19}\right)\end{array}$ & $\begin{array}{c}-6,957 \cdot 10^{-2} \\
(33,92)\end{array}$ \\
\hline$\frac{1}{\sin ^{2} \theta}$ & $\begin{array}{c}1,000 \\
\left(6,550 \cdot 10^{-5}\right)\end{array}$ & $\begin{array}{c}1,000 \\
\left(2,146 \cdot 10^{-6}\right)\end{array}$ & $\begin{array}{c}0,1870 \\
\left(-1,753 \cdot 10^{-8}\right)\end{array}$ & $\begin{array}{c}1,000 \\
\left(9,408 \cdot 10^{-5}\right)\end{array}$ \\
\hline $\cot ^{2} \theta$ & $\begin{array}{c}-1,000 \\
\left(2,430 \cdot 10^{-4}\right)\end{array}$ & $\begin{array}{c}-1,000 \\
\left(6,034 \cdot 10^{-7}\right)\end{array}$ & $\begin{array}{l}-1,180 \cdot 10^{-2} \\
\left(-1,373 \cdot 10^{9}\right)\end{array}$ & $\begin{array}{c}-1,000 \\
\left(3,128 \cdot 10^{-4}\right)\end{array}$ \\
\hline$\frac{1}{(\rho-\tau)^{4} \sin ^{2} \theta}$ & $\begin{array}{l}-0,4142 \\
(111,2)\end{array}$ & $\begin{array}{l}-1,714 \cdot 10^{-4} \\
\left(4,359 \cdot 10^{-5}\right)\end{array}$ & $\begin{array}{l}6,311 \cdot 10^{-12} \\
\left(1,833 \cdot 10^{19}\right)\end{array}$ & $\begin{array}{l}5,784 \cdot 10^{-2} \\
\quad(26,55)\end{array}$ \\
\hline$\frac{1}{(\rho-\tau)^{10 / 3} \sin ^{2} \theta}$ & $\begin{array}{c}-0,2985 \\
(79,94)\end{array}$ & $\begin{array}{l}-2,705 \cdot 10^{-4} \\
\left(8,060 \cdot 10^{-5}\right)\end{array}$ & $\begin{array}{c}8,995 \cdot 10^{12} \\
\left(-1,442 \cdot 10^{19}\right)\end{array}$ & $\begin{array}{c}-2,258 \cdot 10^{-2} \\
(9,089)\end{array}$ \\
\hline$\frac{\cot ^{2} \theta}{\sin ^{2} \theta}$ & $\begin{array}{c}9,974 \cdot 10^{-8} \\
\left(4,800 \cdot 10^{-5}\right) \\
\end{array}$ & $\begin{array}{c}1,732 \cdot 10^{-8} \\
\left(1,324 \cdot 10^{-8}\right)\end{array}$ & 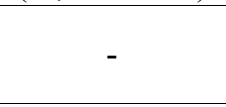 & - \\
\hline$-\frac{1}{(\rho-\tau)^{10 / 3}}$ & - & - & $\begin{array}{l}-1,630 \cdot 10^{-12} \\
\left(-1,618 \cdot 10^{19}\right)\end{array}$ & $\begin{array}{c}2,608 \cdot 10^{-2} \\
(14,21)\end{array}$ \\
\hline$\frac{1}{(\rho-\tau)^{7 / 3}}$ & - & - & $\begin{array}{l}-1,065 \cdot 10^{-10} \\
\left(-5,221 \times 10^{16}\right)\end{array}$ & $\begin{array}{c}6,675 \cdot 10^{-3} \\
(1,866)\end{array}$ \\
\hline$\frac{\cos \theta}{\sin ^{4} \theta}$ & - & - & $\begin{array}{c}-3,160 \cdot 10^{-4} \\
\left(6,635 \cdot 10^{6}\right)\end{array}$ & $\begin{array}{l}5,047 \cdot 10^{-10} \\
\left(1,469 \cdot 10^{-7}\right)\end{array}$ \\
\hline
\end{tabular}

Note. The numeric values in the brackets are, $\sqrt{V(c)}$, the standard errors of $c$. 


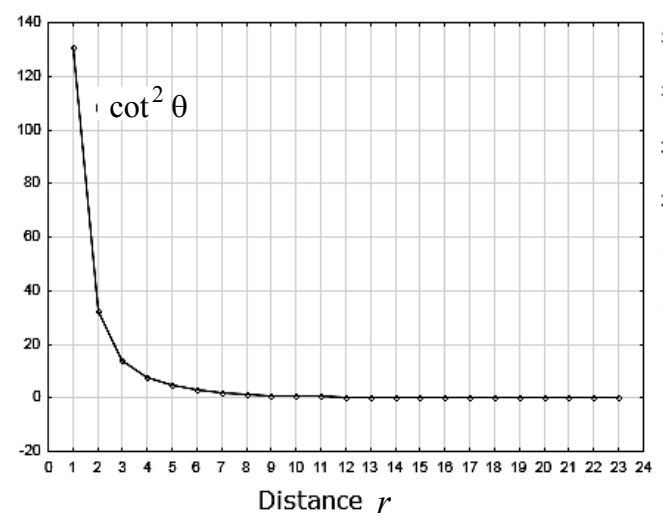

Fig. 5. Components of gravitational field, Fig. 6. Components of gravitational case 1

Note. (Left axis) means that the scale of the function is shown in the left axis; and, (Right axis) in the right axis.

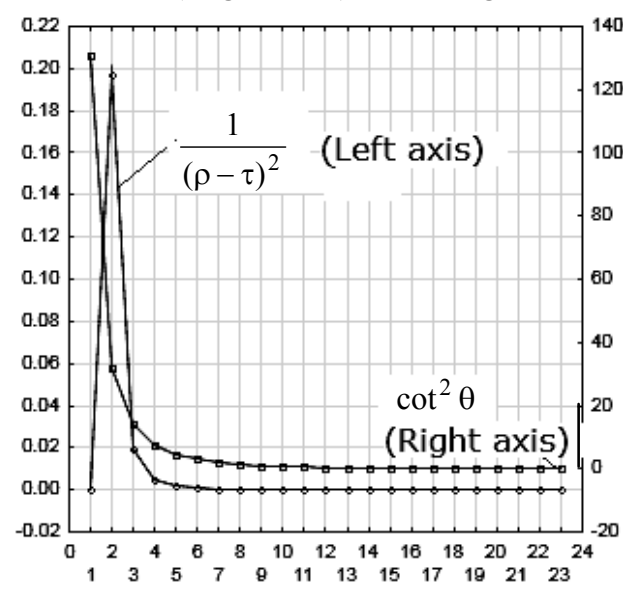

Distance $r$

Fig. 7. Components of gravitational field, Fig. 8. Components of gravitational field, case 2 (2) case 2

\section{CONCLUSION AND RECOMMENDATION}

In this research, we investigated the structure of gravitational field inside of a black hole, assuming that time and space interact each other with $\rho=t+g(r)$, $\tau=t+f(r),(t$ is the time, $r$ is the distance from the center of the black hole, and $f$ and, $g$, are functions of $r$ ). And we also investigated the functions of gravitational waves that are emitted from the inside of the black hole. As the result we found that some of the functions of gravitational waves carry the components of the curvature tensor of gravitational field. It means that we are able to investigate the structure of a black hole by the information carried by the gravitational waves. 
Inside of the black hole the functions shown in Table 1 for the gravitational field and in Table 2 for the gravitational waves, show the same functions: $(\rho-\tau)^{-4}, \cot ^{2} \theta$ and $\sin ^{-2} \theta$. The function of $(\rho-\tau)^{-4}$ in Fig. 6 suggests the presence of a boundary of the black hole, where the gravitational waves are disturbed once before they are moving toward the outside.

On the other hand, if we don't assume the dependence of time and space, the gravitational waves are to be made outside of the black hole, and they do not carry the same functions of the gravitational field. The equations (1) about the gravitational field are $R_{00} \propto r^{-1}, R_{11} \propto r^{-1}, R_{22} \propto r$ and $R_{33} \propto r$; while the equations (2) about the gravitational waves are $r^{-2}, r^{-3}, r^{-4}$ and $r^{-6}$.

In this research, we used the spherical polar coordinate system to describe the curvature of gravitational field, and this system helped us to make numerical simulation possible. However, further mathematical investigations are needed about the curvature tensors.

\section{REFERENCE}

1. Dirac P.A.M. General Theory of Relativity / P.A.M. Dirac. - New York: Florida University, A Wiley Interscience Publication, John Wiley \& Sons, 1975. - 69 p.

2. Goldstein H. Classical Mechanics / H. Goldstein, C.P. Poole, J.L. Safko. $-3^{\text {rd }}$ Edition. - Pearson Education, Inc., 2002. - P. 646 (especially Chapter 7.11 "Introduction to the general theory of relativity"), P. 324-328.

Received 06.01.2020

From the Editorial Board: the article corresponds completely to submitted manuscript. 